\title{
Prospective superficial EPR in-vivo dosimetry study during hypofractionated radiotherapy of breast cancer patients treated with helical tomotherapy
}

\author{
Sebastian Höfel ${ }^{1,2,6^{*}} \mathbb{0}$, Matteo Gandalini' ${ }^{2}$, Michael K. Fix ${ }^{3}$, Malte Drescher ${ }^{1}$ and Felix Zwicker ${ }^{2,4,5}$
}

\begin{abstract}
Background: In-vivo dosimetry (IVD) is a patient specific measure of quality control and safety during radiotherapy. With regard to current reporting thresholds for significant occurrences in radiotherapy defined by German regulatory authorities, the present study examines the clinical feasibility of superficial electron paramagnetic resonance (EPR) IVD of cumulative total doses applied to breast cancer patients treated with helical intensity-modulated radiotherapy (tomotherapy).

Methods: In total, 10 female patients with left- or right-sided breast cancer were enrolled in this prospective IVD study. Each patient received a hypofractionated whole breast irradiation. A total median dose of $42.4 \mathrm{~Gy}$ in 16 fractions ( 5 fractions per week) was prescribed to the planning target volume. The treatments were completely delivered using helical tomotherapy and daily image guidance via megavoltage CT (MVCT). For each patient, three EPR dosimeters were prepared and placed at distinct locations on the patient's skin during the delivery of all fractions. Two dosimeters were placed next to the ipsilateral and contralateral mammilla and one dosimeter was placed ventrally to the thyroid (out-of-primary-beam). The total doses delivered to the dosimeters were readout after all fractions had been administered. The measured total dose values were compared to the planned dose values derived from the treatment planning system (TPS). Daily positional variations (displacement vectors) of the ipsilateral mammilla and of the respective dosimeter were analyzed with respect to the planned positions using the daily registered MVCT image.

Results: Averaged over all patients, the mean absolute dose differences between measured and planned total dose values ( \pm standard deviation (SD)) were: $0.49 \pm 0.85 \mathrm{~Gy}$ for the ipsilateral dosimeter, $0.17 \pm 0.49 \mathrm{~Gy}$ for the contralateral dosimeter and $-0.12 \pm 0.30 \mathrm{~Gy}$ for the thyroid dosimeter. The mean lengths of the ipsilateral displacement vectors ( \pm SD) averaged over all patients and fractions were: $10 \pm 7 \mathrm{~mm}$ for the dosimeter and $8 \pm 4 \mathrm{~mm}$ for the mammilla.

Conclusion: Superficial EPR IVD is suitable as additional safeguard for dose delivery during helical tomotherapy of breast cancer. Despite positional uncertainties in clinical routine, the observed dose deviations at the ipsilateral breast were on average small compared to national reporting thresholds for total dose deviations (i.e. 10\%/4 Gy). EPR IVD may allow for the detection of critical dose errors during whole breast irradiations.
\end{abstract}

*Correspondence: Sebastian.Hoefel@uni-konstanz.de

${ }^{6}$ Department of Chemistry, AG Drescher, University of Konstanz,

Universitätsstraße 10, Box 706, 78457 Konstanz, Germany

Full list of author information is available at the end of the article

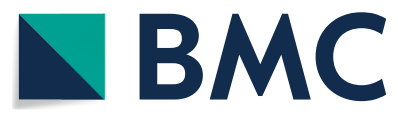

(c) The Author(s) 2021. Open Access This article is licensed under a Creative Commons Attribution 4.0 International License, which permits use, sharing, adaptation, distribution and reproduction in any medium or format, as long as you give appropriate credit to the original author(s) and the source, provide a link to the Creative Commons licence, and indicate if changes were made. The images or other third party material in this article are included in the article's Creative Commons licence, unless indicated otherwise in a credit line to the material. If material is not included in the article's Creative Commons licence and your intended use is not permitted by statutory regulation or exceeds the permitted use, you will need to obtain permission directly from the copyright holder. To view a copy of this licence, visit http://creativecommons.org/licenses/by/4.0/. The Creative Commons Public Domain Dedication waiver (http://creativeco mmons.org/publicdomain/zero/1.0/) applies to the data made available in this article, unless otherwise stated in a credit line to the data. 
Keywords: In vivo, EPR dosimetry, Lithium formate, Alanine, Breast cancer, Radiotherapy, IMRT, Tomotherapy, Hypofractionated

\section{Introduction}

In radiotherapy, tumor control as well as the occurrence of deterministic side effects are, in general, very sensitive to the absorbed dose within the tumor and normal tissue, respectively [1]. Therefore, accuracy requirements of radiation dose delivery to patients are high [1-3].

Each step and component within the applied radiotherapy treatment chain introduce errors and uncertainties that affect the accuracy of the actually delivered dose to the patient [4]. Especially intensity modulated radiotherapy (IMRT) treatments are complex and highly individualized with respect to radiation delivery [5]. IMRT treatment plans are tailored towards optimized organs at risk (OARs) sparing and conformal dose coverage of planning target volumes (PTVs), which often leads to steep dose gradients in the surrounding body regions. The delivered cumulative dose distribution within a patient undergoing fractionated radiotherapy may be different from the planned dose distribution due to anatomical changes during the treatment course (e.g. tissue swelling or shrinkage), intra- and inter-fractional organ motion as well as setup errors of the patient with respect to the treatment beam [6]. Nowadays, image guided radiotherapy (IGRT) is frequently applied to reduce setup errors and by this means to improve the geometric accuracy and uncertainty of dose administration [7]. Moreover, IGRT allows to monitor anatomical changes during the treatment course. However, IGRT is not capable of perfectly reproducing the planned irradiation situation as defined in the treatment plan on each treatment day. Variabilities of the body's outline, daily positional variations of OARs and target volumes etc. need to be tolerated to some extent.

Since recently, a central reporting system for significant occurrences in radiotherapy has been established in Germany $[8,9]$. Severe deviations of the actually delivered cumulative dose from the planned total dose for both, target volumes and OARs may constitute a reportable event. Reporting thresholds are defined in the current radiation protection ordinance [10] and relate to cumulative total doses actually delivered to patients. For target volumes, total dose deviations of $\pm 10 \%$ from the planned mean dose need to be reported. Also local total dose deviations of $\pm 10 \%$ with respect to the planned total dose within target structures are defined as thresholds, if, moreover, the absolute value of the dose difference exceeds 4 Gy. For OARs, reporting thresholds relate to the planned total mean dose and to the total dose constraints defined in the institution's standard operating procedures. Reporting criteria are met, if actually delivered total radiation doses to OARs exceed these values by more than $10 \%$.

In-vivo dosimetry (IVD) is a valuable method for quality assurance in radiotherapy that complements pretreatment quality checks by determining actually delivered radiation doses. So far, point detectors are usually applied during one or a few fractions at representative locations inside body cavities or at the skin level. By this means, IVD is utilized as an additional and independent check of the delivery of single fraction doses to organs at risk and/ or to target volumes [4, 11-13]. Moreover, IVD is used for dose measurements outside the primary beam (outof-field), where clinical treatment planning system (TPS) show accuracy limitations [14]. Although regulatory requirements in Germany imply IVD of cumulative total doses delivered during the complete radiotherapy treatment course, IVD has not yet been routinely applied for this purpose. An appropriate IVD system must meet high accuracy requirements for the determination of cumulative total doses in radiotherapy in order to reliably detect dose errors within the defined reporting thresholds.

Electron paramagnetic resonance (EPR) dosimetry (also known as electron spin resonance (ESR) dosimetry) provides many beneficial features for measuring cumulative total doses in-vivo [15]. While traditionally L-alanine (ALA) was used for EPR dosimetry in radiotherapy [1620], recent studies investigated the applicability of novel detector materials such as lithium formate monohydrate (LFM) in the radiotherapy dose range [21-23]. Compared to ALA, LFM offers higher dose precision $(<3 \%)$ down to doses of around $1 \mathrm{~Gy}$ when applying a practical measuring protocol (10 min readout time per pellet) tailored for routine clinical use in radiotherapy [23]. However, EPR dosimetry and especially EPR IVD is rarely applied in radiotherapy so far.

In a recent proof of principle study, we utilized a rigid anthropomorphic phantom and demonstrated the suitability of superficial EPR IVD using LFM (and ALA) for measuring cumulative total doses during complex head and neck IMRT treatments [24]. Based on the findings of our previous work we concluded that superficial EPR IVD is particularly suitable for total target dose verification, especially when treating near-surface targets. Radiotherapy of the female breast is supposed to be an obvious clinical application example. As a matter of fact, dosimetric treatment verification of whole breast 
irradiations performed with IMRT is of special interest, since inter- and intra-fractional motion such as breathing $[25,26]$, organ swelling or shrinkage [27] as well as variabilities regarding positioning of the patient [25] may affect the actually delivered dose.

The primary aim of the current study was to demonstrate the clinical feasibility of superficial EPR IVD for verifying cumulative total doses delivered during real breast IMRT treatments with regard to current reporting thresholds for significant occurrences. The method is exemplarily demonstrated in the high dose range (next to the PTV), in the intermediate OAR dose range (at the contralateral breast) and in the low dose range (out-ofprimary-beam). Three feasibility aspects are reported: (i) Practicability of superficial dosimeter positioning in clinical routine, (ii) deviations between measured and planned total dose values when dosimeters are exposed to daily positional variations and (iii) the magnitude of these dose deviations in relation to current reporting thresholds.

For this purpose, superficial EPR IVD was performed during 10 real IMRT breast treatments. All patients received a hypofractionated whole breast IMRT treatment using helical tomotherapy and daily image guidance via megavoltage CT (MVCT). Cumulative total doses were measured superficially by placing EPR dosimeters next to the left and right mammillae as well as ventrally to the thyroid (out-of-primary-beam) during all fractions. Each EPR dosimeter consisted of one ALA and one LFM pellet. The measured total dose values were compared to the planned total dose values derived from the TPS dose calculation. Daily positional variations of the ipsilateral mammilla and the respective EPR dosimeter were evaluated using the daily registered MVCT image. Deviations between measured and planned dose values at the dosimeter locations were assessed in the context of current national reporting thresholds for significant occurrences in radiotherapy.

\section{Patients and methods}

\section{Patients and treatment prescription}

Ten female patients diagnosed with left- or right sided, nodal negative breast cancer were enrolled in this in-vivo dosimetry study. All patients underwent breast-conserving surgery and were indicated for adjuvant whole breast radiotherapy. All treatments were performed according to the institution's standard operating procedures. Each patient received a hypofractionated whole breast irradiation using helical tomotherapy and daily IGRT via MVCT imaging. A total median dose of 42.4 Gy in 16 fractions (5 fractions per week) was prescribed to the PTV. The main focus of daily image registration was the correct positioning of the ipsilateral chest wall with respect to the radiation beam. The volume size of the PTV as defined on the planning CT image ranged from 1051 to 2623 ccm. Averaged over all patients, the PTV size was 1596 $\mathrm{ccm}$.

\section{EPR dosimeters and superficial placement}

The EPR dosimeters consisted of a cylindrical polypropylene capsule (outer diameter of $6.4 \mathrm{~mm}$, length of $12 \mathrm{~mm}$ ) containing one ALA and one LFM pellet (see Fig. 1a). The radiation-sensitive pellets had a cylindrical shape with a diameter of $4 \mathrm{~mm}$ and a height of $2 \mathrm{~mm}$ (ALA) or $4 \mathrm{~mm}$ (LFM). Further details regarding dosimeter design and preparation can be found in previous publications $[23,24]$.

For each patient, three EPR dosimeters were prepared and placed at distinct locations on the patient's skin during acquisition of the initial $\mathrm{kV}-\mathrm{CT}$ used for treatment planning (see "Treatment planning" section) and during the delivery of all MVCTs and all treatment fractions. The EPR dosimeters were attached to clear anatomical points of reference: Two EPR dosimeters (labeled as iDos and cDos) were placed next to the ipsilateral and contralateral mammilla (labeled as iMam and cMam), respectively. Quick and easy dosimeter application and removal was realized by using silicone nipple shields (mamivac ${ }^{\circledR}$, $\mathrm{KaWeCo} \mathrm{GmbH}$, Ditzingen, Germany) enclosing the EPR dosimeters (Fig. 1b, c). The third EPR dosimeter was placed in the vicinity of the thyroid, i.e. just below the laryngeal prominence. The thyroid dosimeter (tDos) was attached to the skin using skin-friendly tape (Micropore ${ }^{\mathrm{TM}}$ surgical tape, 3 M Corp., Saint Paul, USA). An example of the dosimeters' placements is shown in Fig. 1d. Great care was taken that the dosimeters' symmetry axis was always in parallel with the $y$-direction of the International Electrotechnical Commission (IEC) accelerator coordinate system displayed in Fig. 1.

Between fractions, the EPR dosimeters were stored in an air-tight box located outside the treatment room. The storage box provided a constant level of $34 \pm 2 \%$ relative humidity in order to minimize possible EPR signal fading over the course of treatment.

\section{Treatment planning}

Computed tomography (CT) datasets were acquired for treatment planning. Image reconstruction was performed with a $2 \mathrm{~mm}$ slice thickness. All patients were positioned in supine position with arms raised above the head. A wedged immobilization system (wingSTEP ${ }^{\mathrm{TM}}$ and MCT wedge evo indexed, IV-T, Innsbruck, Austria) was used in order to increase the reproducibility of patient positioning.

The following OARs were contoured and considered during treatment planning: The left and right lungs, 


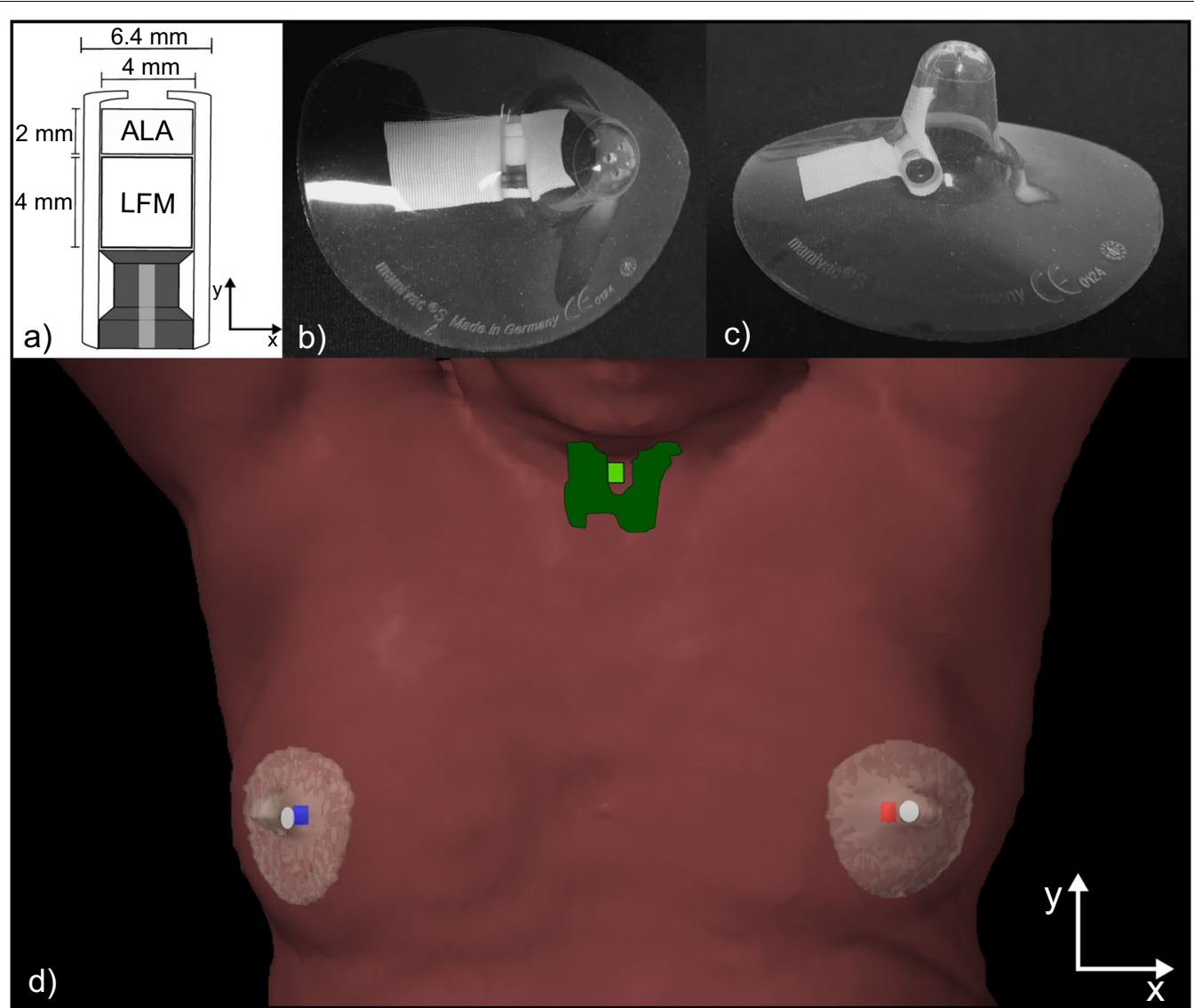

Fig. 1 Sketch of cross-section parallel to the EPR dosimeters' symmetry axis and dimensions of the dosimeter design (a). Pictures of an EPR dosimeter affixed inside the cavity of a nipple shield for quick and easy surface application and removal (b, c). Exemplary 3D view of a left-sided breast cancer patient's body (brown surface) equipped with three EPR dosimeters (d). The ipsilateral EPR dosimeter (iDos) and the contralateral EPR dosimeters (CDos) are shown as red and blue structures, respectively. These dosimeters were placed medial with respect to the left and right mammilla (white structures) using nipple shields (translucent structures). The thyroid and the corresponding EPR dosimeter (tDos) are shown as dark and bright green structures, respectively. Definition of the IEC coordinate system in relation to the dosimeters' geometry and the patient setup $(\mathbf{a}, \mathbf{d})$

the contralateral breast and the heart. The thyroid was contoured as region of interest (ROI) and was located out-of-field. The PTV was defined according to the institution's standard operating procedures; depending on the diagnosis (left/right sided breast cancer), the PTV encompassed the whole left or right mammary gland and always included the chest wall. A safety margin of $2 \mathrm{~cm}$ in cranio-caudal direction was considered during the PTV delineation process. For the purpose of skin sparing, PTV delineation was restricted to a depth of $3 \mathrm{~mm}$ under the skin of the ipsilateral breast. The $3 \mathrm{~mm}$ strip between the PTV and the breast surface was contoured as well $\left(\mathrm{TV}_{\text {Surface }}\right)$ and considered during optimization as separate target volume.

Helical IMRT plans were generated for a Tomotherapy $\mathrm{Hi}-\mathrm{Art}^{\circledR}$ treatment machine by using Accuray's integrated TPS Hi-Art ${ }^{\circledR}$ PlanningStation 5.1.1.6 (Accuray Inc., Sunnyvale, CA, USA). All plans were created with the following settings: Longitudinal field width of $2.5 \mathrm{~cm}$ (dynamic jaw mode $\left(\right.$ TomoEdge $\left.^{\mathrm{TM}}\right)$ ), pitch of 0.282 and planning modulation factors between 2.6 and 2.9. During optimization, plan normalization was set to $\mathrm{D}_{50 \%}(\mathrm{PTV})=42.4$ Gy corresponding to the prescribed total dose. Final dose calculation was performed with a fine dose grid, i.e. the dose voxel size was $2 \mathrm{~mm}$ along all three IEC coordinate axes.

During plan optimization, radiation doses to the OARs were minimized focusing primarily on the dose to the ipsilateral lung while covering at least $99 \%$ of the PTV with a minimum dose of $95 \%$ of the prescribed dose. Dose maxima were restricted to $107 \%$ of the prescribed dose. All treatment plans were clinically acceptable. Based on the TPS final dose calculations, Table 1 summarizes the planned mean doses within the target structures and OARs/ROI for all ten patients included in this study. Figure 2 shows the planned dose distribution in three 
Table 1 Mean doses to the targets and OARs/ROI after final dose calculation for all ten patients together with the arithmetic mean doses ( \pm standard deviation (SD)) over all patients

\begin{tabular}{|c|c|c|c|c|c|c|c|}
\hline \multirow{2}{*}{$\begin{array}{l}\text { Pat \#-right }(R) / \\
\text { left }(L) \text { side }\end{array}$} & \multicolumn{7}{|c|}{ Mean dose to targets and OARs/ROI [Gy] } \\
\hline & PTV & $\mathrm{TV}_{\text {Surface }}$ & $\begin{array}{l}\text { Lung } \\
\text { (ipsilat.) }\end{array}$ & $\begin{array}{l}\text { Lung } \\
\text { (contralat.) }\end{array}$ & Heart & Breast (contralat.) & Thyroid \\
\hline $1-L$ & 42.3 & 37.6 & 9.8 & 3.3 & 5.9 & 3.5 & 1.0 \\
\hline $2-L$ & 42.2 & 37.2 & 11.0 & 4.6 & 6.8 & 5.2 & 1.3 \\
\hline $3-\mathrm{L}$ & 42.2 & 37.4 & 10.1 & 3.9 & 5.6 & 4.6 & 2.9 \\
\hline $4-\mathrm{L}$ & 42.0 & 37.1 & 9.7 & 3.4 & 6.1 & 6.4 & 1.1 \\
\hline $5-R$ & 42.2 & 37.7 & 11.0 & 4.8 & 5.7 & 5.1 & 0.9 \\
\hline $6-R$ & 42.2 & 36.7 & 10.1 & 3.9 & 6.5 & 5.0 & 0.9 \\
\hline 7—L & 42.2 & 37.8 & 11.4 & 4.1 & 5.5 & 6.4 & 2.0 \\
\hline $8-L$ & 42.2 & 37.2 & 11.1 & 4.1 & 7.6 & 5.4 & 1.3 \\
\hline 9-L & 42.2 & 37.3 & 11.4 & 4.6 & 7.5 & 4.2 & 2.6 \\
\hline $10-\mathrm{L}$ & 42.3 & 37.3 & 12.0 & 4.3 & 7.6 & 4.1 & 1.3 \\
\hline Average $( \pm S D)$ & $42.2( \pm 0.1)$ & $37.3( \pm 0.3)$ & $10.8( \pm 0.8)$ & $4.1( \pm 0.5)$ & $6.5( \pm 0.8)$ & $5.0( \pm 0.9)$ & $1.5( \pm 0.7)$ \\
\hline
\end{tabular}

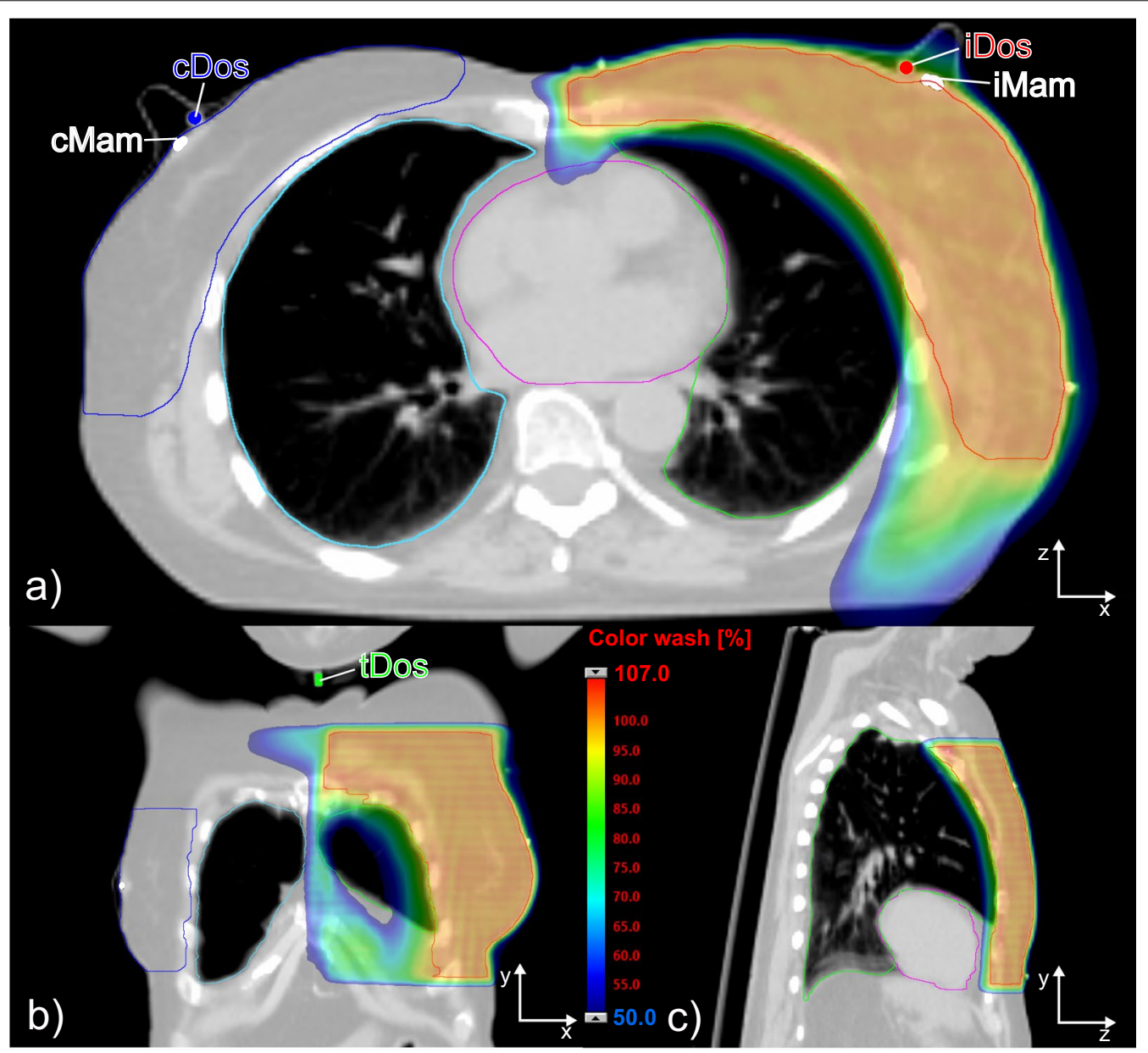

Fig. 2 Axial (a), coronal (b) and sagittal (c) views of the calculated dose distribution superimposed on the planning CT dataset for an exemplary left-sided breast cancer patient (Pat\#10). The ipsilateral dosimeter (iDos) and contralateral dosimeter (cDos) are shown in (a) as red and blue spots, respectively. The right mammilla (cMam) and the left mammilla (iMam) are marked as white segments. The out-of-field location of the thyroid dosimeter (tDos) is illustrated in (b). Contours of the PTV (red) and OARs (ipsilateral lung (green), contralateral lung (cyan), contralateral breast (blue) and heart (purple)) are displayed. The dose distribution is shown in colorwash ranging from 50 to $107 \%$ of the prescribed dose 
orthogonal planes superimposed on the corresponding CT dataset for an exemplary patient. Figure 2 also illustrates the location of the superficially applied EPR dosimeters in relation to the patient's anatomy and to the planned dose distribution.

\section{Superficial dosimetry and uncertainty considerations}

Planned total dose values $D^{p}$ and measured total dose values $D^{m}$ as well as the associated dose uncertainties were obtained according to the procedures reported previously [23, 24]. The main steps are shortly outlined in the following. All uncertainties in this work are determined and expressed in accordance with the Guide to the Expression of Uncertainty in Measurement published by the International Organization for Standardization [28]. Unless otherwise stated, all uncertainties are to be seen as type B standard uncertainties $(1 \sigma)$.

Planned total doses $D^{p}$ to each EPR pellet were derived from the TPS final dose calculations. ALA and LFM pellets were contoured separately with a diameter of $4 \mathrm{~mm}$. HU values inside the pellet contours were taken as received from the planning $\mathrm{CT}$, i.e. no density override was performed. Due to partial volume artefacts resulting from the finite CT slice thickness $(2 \mathrm{~mm})$, two adjacent

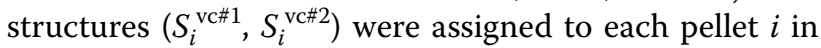
order to capture the pellet's true position (see Fig. 1 in [24]). For each pellet, the mean dose values within these two structures $\left(D_{m}\left(S_{i}{ }^{v c \# 1}\right), D_{m}\left(S_{i}^{v c \# 2}\right)\right)$ were extracted from the dose statistics table of the TPS. Planned dose values $D_{i}^{p}$ for each pellet were defined as the mean value of the mean doses calculated within the two associated structures $\left(D_{i}^{p}=\operatorname{mean}\left\{D_{m}\left(S_{i}^{\nu c \# 1}\right), D_{m}\left(S_{i}^{\nu c \sharp 2}\right)\right\}\right)$. An additional imaging dose due to the clinical IGRT procedure was estimated from phantom measurements (see "IGRT imaging dose consideration" section) and was included in the final planned dose value. The combined uncertainty of the planned dose $u_{c}\left(D^{p}\right)$ consists of two components: The uncertainty of the TPS dose calculation $(2.2 \%$ for iDos (in-field), 5.4\% for cDos and tDos (lying predominantly out of the primary beam)) and an additional contouring uncertainty component specific to each pellet [24].

Measured total dose values $D^{m}$ were obtained via EPR dosimetry. EPR measurements were performed using a compact benchtop spectrometer (MiniScope MS 5000, Magnettech by Freiberg Instruments $\mathrm{GmbH}$, Freiberg, Germany) and a practical dosimetry protocol tailored for routine use in radiotherapy [23]. The EPR pellets were readout after all fractions were delivered. The measurement lasted about $10 \mathrm{~min}$ per pellet. The EPR measurements were corrected for fading and superficial application [24]. Since the EPR dosimeters were irradiated under less controlled conditions $\left(30{ }^{\circ} \mathrm{C} \pm 5{ }^{\circ} \mathrm{C}(2 \sigma)\right.$ assumed for superficial in-vivo application) compared to the dosimeters used for calibration $\left(25^{\circ} \mathrm{C} \pm 2.5^{\circ} \mathrm{C}(2 \sigma)\right)$ an additional temperature correction and uncertainty contribution was considered by applying previously reported correction factors [29]. The combined uncertainty for the measured dose values $u_{c}\left(D^{m}\right)$ consisted of four components: The uncertainties of correcting for fading, for superficial application, for the irradiation temperature and the dose dependent uncertainty of the EPR measurement $[23,24]$.

The combined uncertainty $u_{c}(\Delta D)$ of the dose difference $\Delta D=D^{m}-D^{p}$ is calculated via $u_{c}(\Delta D)^{2}=u_{c}\left(D^{p}\right)^{2}+u_{c}\left(D^{m}\right)^{2}$.

\section{IGRT imaging dose consideration}

Based on the findings of previous research [30] it is assumed that the dose response of the applied EPR dosimeters at the imaging beam quality (about 3.5 MV) equals the dose response at the calibration beam quality (6 MV).

Due to sensitivity limitations of the applied EPR dosimetery system below doses of 1 Gy [23], MVCT imaging doses per fraction were estimated by placing lithium fluoride (LiF) thermoluminescence dosimeters (TLD100) on the surface of tomotherapy's 'cheese phantom'-a cylindrical Virtual Water ${ }^{\mathrm{TM}}$ phantom with a diameter of $30 \mathrm{~cm}$ and a length (IEC $\mathrm{y}$-direction) of $18 \mathrm{~cm}$. The phantom was supposed to represent a human torso and was positioned off-axis, i.e. at IEC coordinates $(x=-2 \mathrm{~cm}$, $\mathrm{z}=-2 \mathrm{~cm}$ ) in relation to the gantry's rotating axis, thus, mimicking a left-sided breast irradiation setup. Six TLDs were attached superficially in the phantom's upper left and upper right quadrants-three TLDs on each side representing possible detector positions of the patient study.

TLDs were provided and read out externally by PTW (PTW GmbH, Freiburg i. Br., Germany). Each of the six TLD detectors consisted of a cylindrical TLD chip (diameter of $4 \mathrm{~mm}$, height of $1 \mathrm{~mm}$ ) that was encapsulated in a cylindrical polymethyl methacrylate (PMMA) rod with a length of $2.5 \mathrm{~cm}$ and a diameter of $5 \mathrm{~mm}$. All TLD chips were exactly located in the center of the PMMA rods. Expressed in polar coordinates $(\mathrm{R}, \phi, \mathrm{Z})$ with the origin lying in the center of the phantom, with $\phi=0^{\circ}$ corresponding to the IEC $\mathrm{z}$ direction and with a $\mathrm{Z}$ axis in parallel with the IEC $y$ axis, the centers of the TLDs were located at: $\left(\mathrm{R}=15.25 \mathrm{~cm}, \phi=\left(-60^{\circ},-50^{\circ},-40^{\circ}, 40^{\circ}, 50^{\circ}\right.\right.$, $\left.\left.60^{\circ}\right), \mathrm{Z}=0 \mathrm{~cm}\right)$.

In the present study, all MVCTs were performed in 'coarse' acquisition mode. In order to improve TLD readout uncertainties, ten 'coarse' MVCTs were applied sequentially to the phantom setup. The determined imaging doses to the TLDs per single MVCT are shown 
in Table 2. The average imaging doses of 1.17 cGy and 0.88 cGy per MVCT were included in the planned dose values ("Superficial dosimetry and uncertainty considerations" section) for the cDos and iDos EPR pellets, respectively.

Table 2 MVCT imaging doses to TLDs placed in the right (R) and left (L) upper quadrant of the 'cheese phantom' for imaging mode'coarse'

\begin{tabular}{ll}
\hline $\begin{array}{l}\text { TLD \# - } \\
\text { (R)/(L) side }\end{array}$ & $\begin{array}{l}\text { Imaging doses to } \\
\text { TLDs per MVCT } \\
{[\mathbf{C G y}]} \\
\text { Coarse mode }\end{array}$ \\
\hline 1-R $\left(-60^{\circ}\right)$ & 1.08 \\
2-R $\left(-50^{\circ}\right)$ & 1.20 \\
3-R $\left(-40^{\circ}\right)$ & 1.24 \\
Average- $R$ & 1.17 \\
4- $\left(40^{\circ}\right)$ & 1.03 \\
5-L $\left(50^{\circ}\right)$ & 0.93 \\
6- $\left(60^{\circ}\right)$ & 0.69 \\
Average- $-\mathrm{L}$ & 0.88 \\
\hline
\end{tabular}

The relative uncertainty of the TLD measurements was stated by PTW as $2.5 \%(1 \sigma)$. We estimated the absolute uncertainty of the total imaging dose (16 fractions) to $u_{\mathrm{MVCT}}=4 \mathrm{mGy}(1 \sigma)$. Compared to other sources of uncertainty $([23,24]$ and Table 3$)$ this contribution is marginal and was therefore neglected.

\section{Positional variations}

Daily absolute positions (IEC coordinates) of the ipsilateral dosimeter $\vec{v} \underset{i \text { Dos }}{\text { frac }}$ and of the ipsilateral mammilla $\vec{v}{ }_{i M a m}^{\text {frac } j}$ in relation to the location on the planning $\operatorname{CT}\left(v_{\text {iDos }}^{\text {Plan }}, \vec{v}\right.$ iMam $)$ were recorded by means of the daily registered MVCT image. Daily

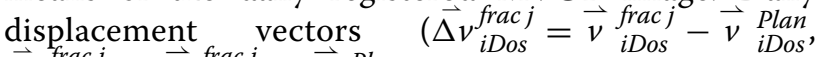
$\vec{\Delta} v_{i \text { Mam }}^{\text {frac } j}=\vec{v}{ }_{i \text { Mam }}^{\text {fracj }}-\vec{v}{ }_{i \text { Mam }}^{\text {Plan }}$ ) were determined for all treatment fractions $j$.

The contralateral dosimeter (cDos) and contralateral mammilla (cMam) as well as the thyroid and the corresponding dosimeter (tDos) were located outside the MVCT's field-of-view.

Table 3 Measured and planned total doses to ALA and LFM pellets for all patients

\begin{tabular}{|c|c|c|c|c|c|c|}
\hline \multirow[t]{2}{*}{ Pat \# } & \multicolumn{2}{|l|}{ iDos } & \multicolumn{2}{|l|}{ cDos } & \multicolumn{2}{|l|}{ tDos } \\
\hline & $D^{m}\left(u_{c}\right)$ & $D^{p}\left(u_{c}\right)$ & $D^{m}\left(u_{c}\right)$ & $D^{p}\left(u_{c}\right)$ & $D^{m}\left(u_{c}\right)$ & $D^{p}\left(u_{c}\right)$ \\
\hline & \multicolumn{6}{|c|}{ Total doses to ALA pellets [Gy] } \\
\hline 1 & $44.79(0.34)$ & $45.00(1.02)$ & $3.24(0.13)$ & $3.02(0.15)$ & $1.24(0.13)$ & $1.14(0.06)$ \\
\hline 2 & $44.00(0.33)$ & $43.58(0.97)$ & $6.77(0.14)$ & $5.99(0.32)$ & $1.55(0.13)$ & $1.14(0.06)$ \\
\hline 3 & $41.52(0.32)$ & $40.38(0.91)$ & $5.08(0.13)$ & $5.33(0.27)$ & $1.06(0.13)$ & $1.45(0.08)$ \\
\hline 4 & $41.91(0.32)$ & $40.92(0.90)$ & $7.75(0.14)$ & $7.23(0.38)$ & $1.23(0.13)$ & $1.27(0.07)$ \\
\hline 5 & $40.17(0.31)$ & $38.47(0.96)$ & $5.06(0.13)$ & $4.50(0.23)$ & $1.33(0.13)$ & $1.04(0.06)$ \\
\hline 6 & $38.77(0.30)$ & $38.68(0.85)$ & $6.69(0.14)$ & $6.26(0.33)$ & $1.04(0.13)$ & $1.20(0.07)$ \\
\hline 7 & $38.40(0.30)$ & $36.30(1.00)$ & $6.19(0.14)$ & $5.76(0.30)$ & $1.17(0.13)$ & $1.12(0.06)$ \\
\hline 8 & $38.48(0.30)$ & $38.70(0.85)$ & $4.93(0.13)$ & $4.62(0.24)$ & $1.26(0.13)$ & $1.84(0.10)$ \\
\hline 9 & $41.26(0.32)$ & $40.67(0.98)$ & $2.73(0.13)$ & $3.61(0.18)$ & $1.72(0.13)$ & $1.47(0.09)$ \\
\hline \multirow[t]{2}{*}{10} & $41.39(0.32)$ & $41.09(1.05)$ & $3.77(0.13)$ & $3.51(0.20)$ & $1.04(0.13)$ & $1.22(0.07)$ \\
\hline & \multicolumn{6}{|c|}{ Total doses to LFM pellets [Gy] } \\
\hline 1 & $44.45(0.42)$ & $44.97(0.99)$ & $3.11(0.05)$ & $3.01(0.15)$ & $1.23(0.04)$ & $1.22(0.07)$ \\
\hline 2 & $43.53(0.41)$ & $43.63(0.97)$ & $6.53(0.07)$ & $5.96(0.31)$ & $1.50(0.04)$ & $1.21(0.07)$ \\
\hline 3 & $41.33(0.39)$ & $40.66(0.89)$ & $5.10(0.06)$ & $5.35(0.28)$ & $1.15(0.03)$ & $1.51(0.08)$ \\
\hline 4 & $41.61(0.40)$ & $40.26(0.94)$ & $7.50(0.08)$ & $7.17(0.39)$ & $1.03(0.03)$ & $1.35(0.07)$ \\
\hline 5 & $39.19(0.37)$ & $38.26(0.87)$ & $4.97(0.06)$ & $4.37(0.23)$ & $0.95(0.03)$ & $1.15(0.09)$ \\
\hline 6 & $38.83(0.37)$ & $38.57(0.85)$ & $6.72(0.07)$ & $6.36(0.36)$ & $0.97(0.03)$ & $1.27(0.07)$ \\
\hline 7 & $38.75(0.37)$ & $36.87(0.81)$ & $6.19(0.07)$ & $5.76(0.30)$ & $1.04(0.03)$ & $1.19(0.07)$ \\
\hline 8 & $38.41(0.37)$ & $38.80(0.85)$ & $4.67(0.06)$ & $4.68(0.24)$ & $1.29(0.04)$ & $2.01(0.13)$ \\
\hline 9 & $41.08(0.39)$ & $41.06(0.91)$ & $2.56(0.04)$ & $3.73(0.19)$ & $1.49(0.04)$ & $1.58(0.09)$ \\
\hline 10 & $40.87(0.39)$ & $41.98(0.93)$ & $3.67(0.05)$ & $3.54(0.18)$ & $1.04(0.03)$ & $1.30(0.07)$ \\
\hline
\end{tabular}

Absolute combined uncertainties $(1 \sigma)$ are given in brackets 


\section{Results}

\section{Practicability}

From a clinical staff's perspective, daily application of the EPR dosimeters on the patients' skin was quick, simple and reproducible, due to the positioning aids used (nipple shields, skin-friendly tape; see "EPR dosimeters and superficial placement" section) and due to clear anatomical points of reference (left/right mammilla, laryngeal prominence), respectively. Superficial dosimeter application was well tolerated by the patients.

\section{Positional variations}

For the ipsilateral mammilla (iMam) and the respective dosimeter (iDos), positional variations with respect to the planned situation were recorded on the basis of the daily registered MVCT images. Figure 3 shows the residual (after applying IGRT patient setup corrections) mean displacement values in IEC $\mathrm{x}_{-}, \mathrm{y}_{-}$, and $\mathrm{z}$ - direction as well as the mean length of the displacement vector $\overrightarrow{|\overrightarrow{\Delta v}|}$ averaged over all treatment fractions for each patient. Averaged over all patients and fractions, the mean lengths of the ipsilateral displacement vectors ( \pm standard deviation (SD)) were: $10 \pm 7 \mathrm{~mm}$ for iDos and $8 \pm 4 \mathrm{~mm}$ for iMam.

The distance (IEC $y$ - component) of the center of the thyroid dosimeter (tDos) to the cranial PTV border was determined on the planning $\mathrm{CT}$ image and ranged from $2.0 \mathrm{~cm}$ (Pat \#8) to $5.0 \mathrm{~cm}$ (Pat \#5). Averaged over all patients, the mean distance $( \pm \mathrm{SD})$ was $3.8 \pm 0.8 \mathrm{~cm}$.

\section{Total dose deviations}

Measured $\left(D^{m}\right)$ and planned $\left(D^{p}\right)$ total dose values to the ALA and LFM pellets in combination with absolute combined uncertainties (see "Superficial dosimetry and uncertainty considerations" section) are listed in Table 3 for all three dosimeter locations (iDos, cDos, tDos) and for all patients. Differences between measured and planned dose values observed at each dosimeter location are illustrated in Fig. 4. The dose differences $\Delta D=D^{m}-D^{p}$ are shown in absolute as well as relative terms with respect to the planned dose. Uncertainty margins $(1 \sigma)$ for the dose difference are indicated by the error bars.
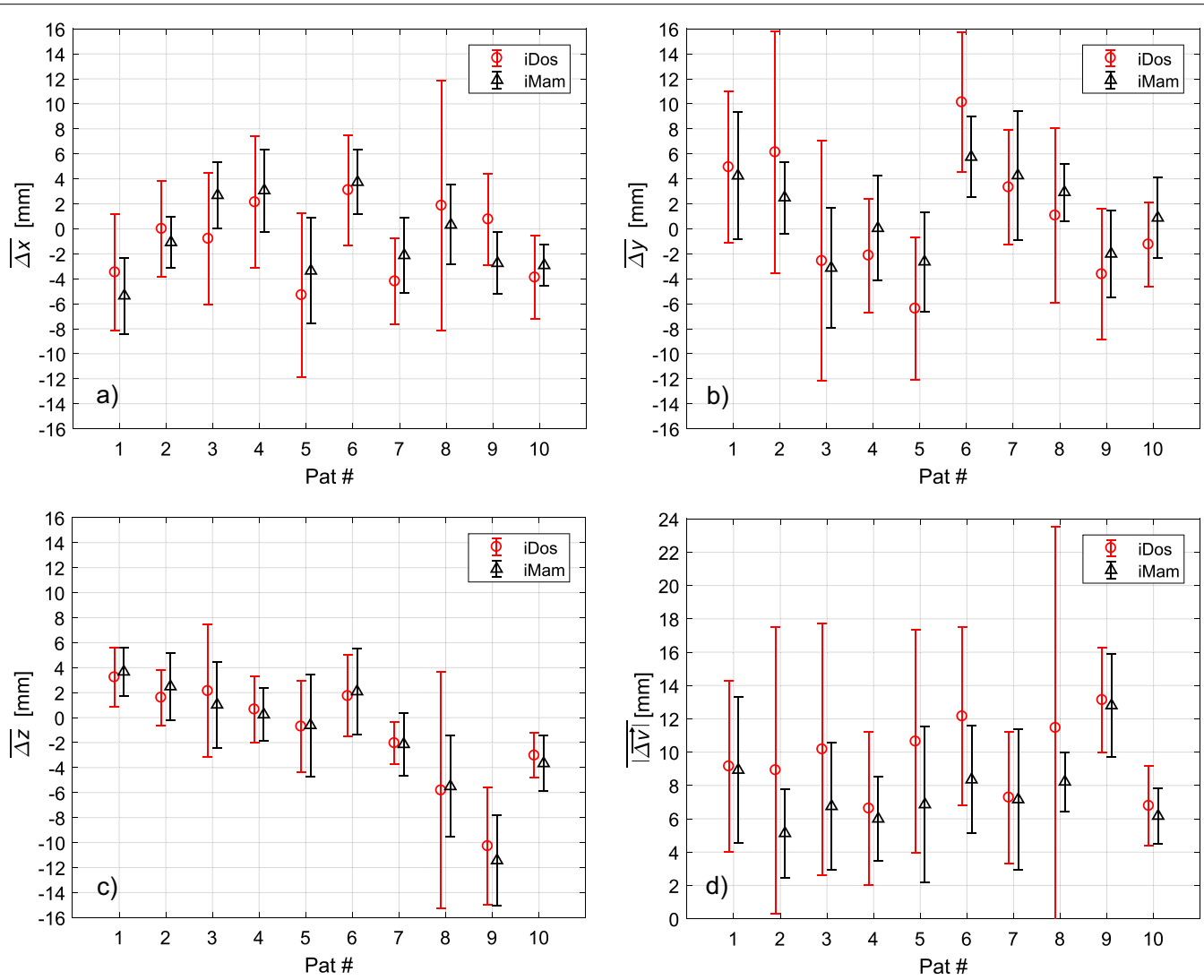

Fig. 3 Mean IEC $x$ - (a), $y$ - (b) and z-component (c) of the displacement vectors $\vec{\Delta} \mathrm{v}$ for iDos (red circles) and for iMam (black triangles) over all fractions and for each patient. Standard deviations (SD) observed over all fractions are indicated by the error bars. Mean lengths of the displacement vector $\overline{|\overrightarrow{\Delta v}|}( \pm \mathrm{SD})$ over all fraction are shown in $(\mathbf{d})$ for each patient 

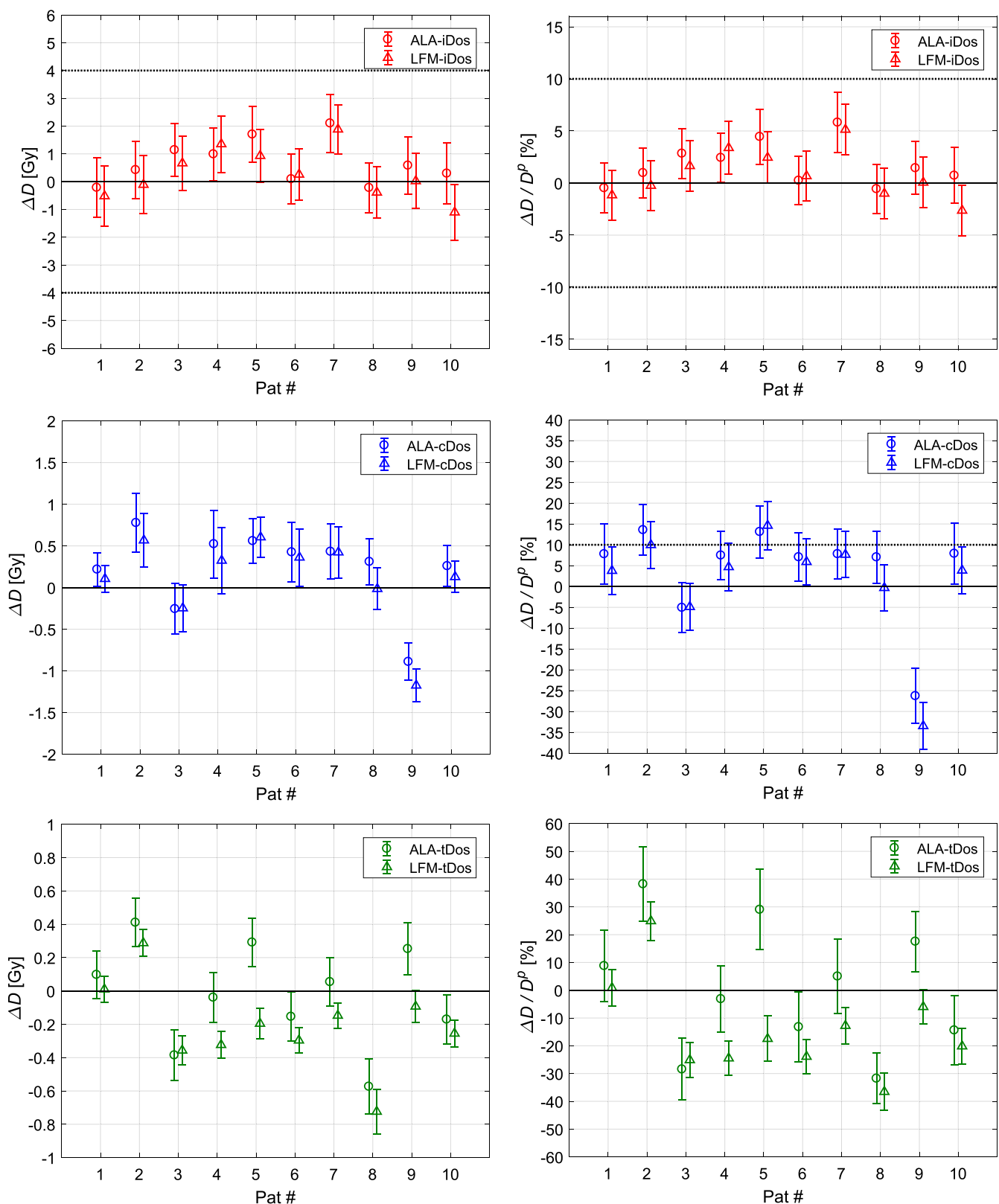

Fig. 4 Absolute $\triangle D$ (left) and relative $\triangle D / D^{p}$ (right) dose differences between measured $D^{m}$ and planned $D^{p}$ total dose values for all patients at EPR dosimeter locations: iDos (top), cDos (middle) and tDos (bottom). For comparison, the results obtained via ALA (circles) and via LFM (triangles) are grouped together. Combined uncertainties $(1 \sigma)$ of the dose difference are represented by error bars. Reporting thresholds (10\%/4 Gy) are indicated by the black dotted lines where applicable

The measured total doses to the EPR dosimeters $( \pm \mathrm{SD})$ averaged over all patients and both pellet types were $40.9 \pm 2.1$ Gy for iDos, $5.2 \pm 1.6$ Gy for cDos and $1.2 \pm 0.2$ Gy for tDos. The observed dose deviations were in the following ranges: From $-1.1 \mathrm{~Gy}$ to $2.1 \mathrm{~Gy}$ for iDos, from -1.2 Gy to 0.8 Gy for cDos and from -0.7 Gy to 0.4 Gy for tDos.
Averaged over all patients and both EPR pellet types, the mean absolute dose differences between measured and planned dose values $( \pm \mathrm{SD})$ were: $0.49 \pm 0.85$ Gy for the ipsilateral dosimeter, $0.17 \pm 0.49 \mathrm{~Gy}$ for the contralateral dosimeter and $-0.12 \pm 0.30$ Gy for the thyroid dosimeter. 


\section{Comparison to reporting thresholds}

Dose deviations between measured and planned total dose values were overall small for the ipsilateral dosimeter (iDos) compared to the $10 \% / 4$ Gy criterion (Fig. 4).

For the contralateral dosimeter, three out of 20 EPR measurements (Pat\#2 and Pat\#5) exceeded the planned dose values by more than $10 \%$ while the error bars for the dose difference still covered the threshold limit.

\section{Discussion}

In the present prospective IVD study, EPR dosimeters were applied superficially in order to monitor and verify total dose delivery at target, OAR and ROI locations during helical tomotherapy of ten female breast cancer patients, each receiving a complete course of hypofractionated whole breast radiotherapy.

Compared to TLD in-vivo dosimetry [25], EPR dosimetry is suited for measuring total radiation doses accumulated over a complete treatment course. Low signal fading rates, non-destructive readout and highly water equivalent dosimeter materials with negligible dependency on beam quality, dose rate and angle of beam incidence [15] are key features of EPR dosimetry, thus, being suitable for in-vivo application. However, reported EPR IVD studies on patients are rare. EPR IVD using ALA was first reported during total body irradiations $[16,31]$ and shortly thereafter also in brachytherapy $[17,18]$. More recently, ALA dosimetry was performed in body cavities during prostate [19] and gynecological [32] external beam radiotherapy (EBRT). Superficial EPR IVD of OAR doses using ALA dosimeters during volumetric modulated arc therapy (VMAT) of breast cancer patients was shown by Wagner et al. [20]. In their study, the EPR dosimeters were solely applied to the contralateral breast. Thus, doses to the EPR dosimeters were between 3 and $20 \mathrm{~Gy}$, i.e. less than $50 \%$ of the prescribed target dose. In the present study, a novel EPR dosimetry material (LFM) was applied during breast EBRT. Besides, ALA pellets were used for comparison purposes. The EPR dosimeters were tested in a wide dose range: close to the PTV receiving high doses in the order of the prescribed dose, on the contralateral breast (intermediate dose) and in front of the thyroid lying out-of-primary-beam (low dose). To our knowledge, in vivo application of LFM dosimeters during patient treatments has not been reported so far. The main advantage of using LFM dosimeters in vivo is their increased sensitivity at lower doses [21-23]. This can be seen from the reduced error bars for the thyroid dosimeter (tDos) in Fig. 4. For tDos, the relative uncertainties $(1 \sigma)$ of the observed dose differences averaged over all patients were 6.6\% (LFM) and 12.2\% (ALA). Although in this study the EPR dosimeters were read out only once after all fractions were delivered, earlier and repetitive evaluations during the treatment course are basically possible with EPR dosimetry since the dose read-out is non-destructive. By this means, dosimetric verification of target and OAR doses is expected to be feasible also between fractions. In these situations, i.e. at lower doses, LFM dosimeters may show better performance at target and OAR locations compared to ALA.

In the present study, daily image registrations were performed on the ipsilateral chest wall provoking positional variations of the ipsilateral mammilla (iMam) and dosimeter (iDos) with respect to the planned situation (Fig. 3). Despite these positional variations during the course of treatment, total dose differences between measured and planned dose values for the ipsilateral dosimeter (iDos) were below 2.1 Gy (Table 3, Fig. 4). Of course, this claim is subject to the magnitude of positional variabilities. Therefore, positional variations of the ipsilateral dosimeter and of the ipsilateral mammilla were recorded in order to provide a quantitative side condition. Daily positional displacements of the ipsilateral mammilla with respect to the planned position were in the order of $8 \pm 4 \mathrm{~mm}$ for the IGRT procedure followed in this study (Fig. 3). Similar displacement values were observed for the respective EPR dosimeter (see mean values for iDos and iMam in Fig. 3), however, daily positioning of the dosimeter on the patients' skin introduced an additional variability leading to increased standard deviations (compare SD values for iDos and iMam in Fig. 3). No significant correlation between the observed dose deviations $\Delta D$ for iDos and any of the Cartesian components or the length of the recorded mean displacement vector for iDos was found. Pearson correlation coefficients were below 0.3 and corresponding p-values were all above 0.2 .

The black dotted horizontal lines in Fig. 4 indicate current reporting thresholds when reporting criteria are translated to the planned total dose values for the EPR dosimeters.

All treatments were performed regularly without special incidents. As a consequence, the results suggest that the observed dose deviations (Fig. 4) are due to daily positional variations and anatomical changes on the one hand and due to dose uncertainties on the other hand.

For the target dosimeter (iDos), the dose deviations and uncertainties were small compared to the reporting thresholds (10\%/4 Gy) featuring EPR IVD as a robust technique for critical dose error detection. Overall, total dose deviations detected by EPR dosimetry were mostly within reporting limits. Exceptions occurred for Pat\#2 and Pat\#5 at the contralateral breast (cDos). Averaged over all patients, however, the relative dose difference $\Delta D / D^{p}$ observed for cDos was $4.0 \%$ (ALA) and $1.7 \%$ (LFM). 
The relative uncertainties $(1 \sigma)$ of the observed dose differences averaged over all patients were $2.4 \%$ (LFM) and 2.5\% (ALA) for iDos and 5.6\% (LFM) and 6.3\% (ALA) for cDos, i.e. smaller than the translated reporting threshold of $10 \%$ of the planned dosimeter dose.

Absolute total dose deviations for the contralateral dosimeter (cDos) were mostly in the range of $\pm 1 \mathrm{~Gy}$. Daily positional variations of the contralateral dosimeter and the contralateral mammilla could not be recorded, since the PTV was positioned as close as possible to the machine isocenter during treatment planning and, thus, the daily locations of cDos and cMam were outside the MVCTs field of view. In a previous study it was demonstrated that the applied dose calculation algorithm may underestimate doses to OAR lying predominantly out-of-primary-beam incidence [24]. However, we suppose that the dose deviations observed in this study are rather due to positional uncertainties of the contralateral breast. This assumption is supported by the fact that the negative outliers (Fig. 4, middle) are observed for patients with the largest PTVs (Pat \#3: $2623 \mathrm{ccm}$ and Pat \#9: 2413 $\mathrm{ccm})$, where positional variations are likely more pronounced. The mean PTV size $( \pm \mathrm{SD})$ among the remaining eight patients was $1365 \pm 260 \mathrm{ccm}$. We suppose that the observed dose deviations for cDos could have been improved by reducing setup errors of the contralateral breast.

Dose measurements next to the thyroid were performed in order to demonstrate the out-of-field applicability of EPR IVD during radiotherapy treatments. The results show that EPR IVD is capable of measuring low cumulative dose values out-of-primary-beam in the order of 1-2 Gy. However, absolute dose differences $\Delta D$ between -0.7 Gy and 0.4 Gy were observed. Relative dose differences $\Delta D / D_{p}$ were between $\pm 40 \%$ (Fig. 4) with a relative uncertainty in the order of $6.6 \%$ (LFM) and $12.2 \%$ (ALA). Daily positional variations of the tDos dosimeter could not be recorded since its position was located outside the daily MVCT scan. It is presumed that the observed dose differences were mainly caused by positional variations (neck setup and $\mathrm{tDos}$ positioning). Averaged over all patients, the mean absolute dose differences $( \pm \mathrm{SD})$ was $-0.12 \pm 0.30 \mathrm{~Gy}$, i.e. the scattering of the dose differences is too large and the sample size too small to assess any systematic errors.

The present study demonstrates the clinical feasibility of superficial EPR IVD of total doses delivered during breast cancer treatments. For the ipsilateral dosimeter locations considered in this work, the observed dose differences between measured and planned total doses to the EPR dosimeters were small compared to reporting thresholds. High cumulative total doses (around $40 \mathrm{~Gy}$ ) at the ipsilateral breast as well as intermediate and lower total doses (between 1 and 6 Gy) at OAR and ROI locations could be measured. A practical EPR dosimetry system tailored for routine clinical use was applied featuring a read out time of 10 min per pellet. The performances of ALA and LFM pellets were comparable in this study. Although not explicitly shown in this study, LFM is expected to be superior for IVD at lower doses (e.g. when measuring single fraction doses at target or OAR locations) due to increased dose precision compared to ALA [23].

\section{Conclusion}

Despite remaining positional uncertainties during imageguided helical tomotherapy of breast cancer, the dose differences between planned and measured cumulative total doses obtained via superficial EPR IVD as well as combined uncertainties of the dose differences were small for the ipsilateral dosimeter compared to current reporting thresholds in radiotherapy. Thus, EPR IVD is suitable and clinically feasible to assist in detecting, preventing and investigating severe dose misadministration to the treated breast according to current reporting criteria. Dose delivery to the contralateral breast and to the thyroid lying out-of-field could be monitored down to approximately 1 Gy cumulative dose. Superficial EPR IVD is to be seen as an additional safeguard for monitoring cumulative total doses to radiotherapy patients. In future clinical routine, superficial EPR IVD could assist in recognizing treatment errors and may support further investigations whether the criteria for reporting are met.

\section{Abbreviations \\ ALA:: L-Alanine; cDos:: Contraleteral dosimeter; cMam:: Contralateral Mam- milla; CT.: Computed tomography; EPR:: Electron paramagnetic resonance; IEC:: International Electrotechnical Commission; iDos:.: Ipsilateral dosimeter; IGRT:: Image guided radiotherapy; iMam:: I psilateral mammilla; IMRT:: Intensity modulated radiotherapy; IVD:: In vivo dosimetry; LFM:: Lithium formate mono- hydrate; LiF:: Lithium fluoride; MVCT:: Megavoltage computed tomography; TLD:: Thermoluminescence dosimeter; OAR:: Organ at risk; PMMA:: Polymethyl methacrylate; PTV:: Planning target volume; ROI:: Region of interest; SD:: Stand- ard deviation; tDos:: Thyroid dosimeter; TPS:: Treatment planning system.}

\begin{abstract}
Acknowledgements
The authors would like to thank our clinical staff members for their high level of motivation, careful dosimeter handling and for their compliance with the study protocol. The following people were actively involved and contributed largely to the success of the present study: Dr. med. Sylke Liebig, Dr. med. Peter Köhler, Louisa Deeg, MSc. Corinna Kirchner, MSc. Michael Schempp, Dipl.-Phys. Tanja Hertel, Elfi Streit, Tatjana Hofer, Dagmar Damoune, Petra Haas, Claudia Baumhof, Melis Kilic and Lena Homann. Special thanks to MSc. Corinna Kirchner for her assistance during dosimeter preparation and to MSc. Pauline Liebig for her support during data evaluation.
\end{abstract}

\section{Authors' contributions}

SH prepared the dosimeters, performed EPR measurements, collected and evaluated the data and wrote the manuscript. MG assisted in the EPR experiments and was involved in the study design, treatment planning and patient education. MKF was involved in the study design, data interpretation and data visualization. MD organized and supervised EPR experiments 
and was involved in EPR data analysis. FZ initiated and organized the clinical implementation of EPR in vivo dosimetry, he was involved in the study design, treatment planning, patient education and data interpretation. All authors read and approved the final manuscript.

\section{Funding}

Open Access funding enabled and organized by Projekt DEAL.

\section{Availability of data and materials}

All original data will be made available upon reasonable request.

\section{Declarations}

\section{Ethical approval and consent to participate}

The study was conducted according to the World Medical Association Declaration of Helsinki and the ICMJE Recommendations for the Protection of Research Participants and approved by the ethics committee of the Landesärztekammer Baden-Württemberg, Stuttgart, Germany (AZ: F-2018-100).

\section{Consent for publication}

All authors provided their consent for publication.

\section{Competing interests}

All authors disclose any potential conflict of interest.

\section{Author details}

${ }^{1}$ Department of Chemistry and Konstanz Research School Chemical Biology, University of Konstanz, Konstanz, Germany. ${ }^{2}$ Klinik und Praxis für Strahlentherapie am Klinikum Konstanz, Konstanz, Germany. ${ }^{3}$ Division of Medical Radiation Physics and Department of Radiation Oncology, Inselspital, Bern University Hospital and University of Bern, Bern, Switzerland. ${ }^{4}$ Department of Radiation Oncology, Heidelberg University Hospital, Heidelberg, Germany. ${ }^{5}$ Clinical Cooperation Unit Molecular Radiation Oncology, German Cancer Research Center (DKFZ), Heidelberg, Germany. ${ }^{6}$ Department of Chemistry, AG Drescher, University of Konstanz, Universitätsstraße 10, Box 706, 78457 Konstanz, Germany.

\section{Received: 29 June 2021 Accepted: 25 October 2021}

Published online: 30 October 2021

\section{References}

1. Brahme A. Dosimetric precision requirements in radiation therapy. Acta Radiol Oncol. 1984;23:379-91. https://doi.org/10.3109/028418684091360 37.

2. International Commission on Radiation Units and Measurements (ICRU) Report 24, J ICRU. os13 (1976) NP-NP. https://doi.org/10.1093/jicru/os13.1. Report24.

3. Mijnheer BJ, Battermann JJ, Wambersie A. What degree of accuracy is required and can be achieved in photon and neutron therapy? Radiother Oncol. 1987;8:237-52. https://doi.org/10.1016/S0167-8140(87)80247-5.

4. International Atomic Energy Agency. Accuracy requirements and uncertainties in radiotherapy. Human Health Series No. 31, IAEA, Vienna (2016).

5. Cho B. Intensity-modulated radiation therapy: a review with a physics perspective. Radiat Oncol J. 2018;36:1-10. https://doi.org/10.3857/roj. 2018.00122.

6. Bortfeld T, Jiang SB, Rietzel E. Effects of motion on the total dose distribution. Semin Radiat Oncol. 2004;14:41-51. https://doi.org/10.1053/j.semra donc.2003.10.011.

7. Dawson LA, Sharpe MB. Image-guided radiotherapy: rationale, benefits, and limitations. Lancet Oncol. 2006;7:848-58. https://doi.org/10.1016/ S1470-2045(06)70904-4.

8. Gesetz zum Schutz vor der schädlichen Wirkung ionisierender Strahlung vom 27. Juni 2017 (BGBI. 2017IS. 1966).

9. Brix G, Griebel J, Czarwinski R. Melde- und Informationssystem für bedeutsame Vorkommnisse bei Strahlenanwendungen in der Medizin: Struktur. Zuständigkeiten und Meldekriterien, Zeitschrift Für Medizinische Physik. 2019;29:66-76. https://doi.org/10.1016/j.zemedi.2018.11.003.
10. Verordnung zum Schutz vor der schädlichen Wirkung ionisierender Strahlung vom 29. November 2018 (BGBI. 2018 I S. 2034, 2036)

11. International Commission on Radiological Protection. Prevention of accidents to patients undergoing radiation therapy. ICRP Publication 86, ICRP, Bethesda, MD (2000).

12. International Atomic Energy Agency. Development of procedures for in vivo dosimetry in radiotherapy. Human Health Reports No. 8, IAEA, Vienna (2013).

13. Van Dam J, Marinello G. Methods for in vivo dosimetry in external radiotherapy, ESTRO Booklet No. 1. 2nd ed. Brussels: European Society for Radiation Oncology, ESTRO; 2006.

14. Schneider U, Hälg RA, Hartmann M, Mack A, Storelli F, Joosten A, Möckli R, Besserer J. Accuracy of out-of-field dose calculation of tomotherapy and cyberknife treatment planning systems: a dosimetric study. Z Med Phys. 2014;24:211-5. https://doi.org/10.1016/j.zemedi.2013.10.008.

15. Baffa $\mathrm{O}$, Kinoshita A. Clinical applications of alanine/electron spin resonance dosimetry. Radiat Environ Biophys. 2014;53:233-40. https://doi. org/10.1007/s00411-013-0509-2.

16. Indovina PL, Benassi M, Giacco GC, Primavera A, Rosati A. In vivo ESR dosimetry in total body irradiation. Strahlenther Onkol. 1989;165:611-6.

17. Kuntz F, Pabst JY, Delpech JP, Wagner JP, Marchioni E. Alanine-ESR in vivo dosimetry: a feasibility study and possible applications. Appl Radiat Isot. 1996;47:1183-8.

18. Schaeken B, Scalliet P. One year of experience with alanine dosimetry in radiotherapy. Appl Radiat Isot. 1996;47:1177-82. https://doi.org/10.1016/ S0969-8043(96)00040-1.

19. Wagner D, Anton M, Vorwerk H, Gsänger T, Christiansen H, Poppe B, Hess CF, Hermann RM. In vivo alanine/electron spin resonance (ESR) dosimetry in radiotherapy of prostate cancer: a feasibility study. Radiother Oncol. 2008;88:140-7. https://doi.org/10.1016/j.radonc.2008.03.017.

20. Wagner DM, Hüttenrauch P, Anton M, von Voigts-Rhetz P, Zink K, Wolff HA. Feasibility study of entrance and exit dose measurements at the contra lateral breast with alanine/electron spin resonance dosimetry in volumetric modulated radiotherapy of breast cancer. Phys Med Biol. 2017;62:5462-72. https://doi.org/10.1088/1361-6560/aa6ee2.

21. Gustafsson $H$, Lund E, Olsson S. Lithium formate EPR dosimetry for verifications of planned dose distributions prior to intensity-modulated radiation therapy. Phys Med Biol. 2008;53:4667. https://doi.org/10.1088/ 0031-9155/53/17/014.

22. Adolfsson E, Gustafsson H, Lund E, AlmCarlsson G, Isson SO, CarlssonTedgren $\AA$. A system for remote dosimetry audit of 3D-CRT, IMRT and VMAT based on lithium formate dosimetry. Radiother Oncol. 2014;113:279-82. https://doi.org/10.1016/j.radonc.2014.11.027.

23. Höfel S, Stehle M, Zwicker F, Fix MK, Drescher M. A practical EPR dosimetry system for routine use in radiotherapy: uncertainty analysis of lithium formate dosimeters at the therapeutic dose level. Phys Med Biol. 2021;66: 045005. https://doi.org/10.1088/1361-6560/ab9414.

24. Höfel S, Fix MK, Drescher M, Zwicker F. Suitability of superficial Electron Paramagnetic Resonance dosimetry for in-vivo measurement and verification of cumulative total doses during IMRT: a proof of principle. Z Med Phys. 2021. https://doi.org/10.1016/j.zemedi.2021.03.006.

25. Zibold F, Sterzing F, Sroka-Perez G, Schubert K, Wagenknecht K, Major G, Debus J, Herfarth K. Surface dose in the treatment of breast cancer with helical tomotherapy. Strahlenther Onkol. 2009;185:574-81. https://doi. org/10.1007/s00066-009-1979-7.

26. Moeckly SR, Lamba M, Elson HR. Respiratory motion effects on whole breast helical tomotherapy. Med Phys. 2008;35:1464-75. https://doi.org/ 10.1118/1.2841936

27. Klepper R, Höfel S, Botha U, Köhler P, Zwicker F. Dosimetric effects of swelling or shrinking tissue during helical tomotherapy breast irradiation. A phantom study. J Appl Clin Med Phys. 2014;15:382-91. https://doi.org/ 10.1120/jacmp.v15i4.4873.

28. BIPM, IEC, IFCC, ILAC, ISO, IUPAC, IUPAP and OIML, Evaluation of measurement data-guide to the expression of uncertainty in measurement JCGM 100:2008. https://www.bipm.org/utils/common/documents/jcgm/ JCGM_100_2008_E.pdf.

29. Waldeland E, Helt-Hansen J, Malinen E. Characterization of lithium formate EPR dosimeters for high dose applications-Comparison with alanine. Radiat Meas. 2011;46:213-8. https://doi.org/10.1016/j.radmeas. 2010.11.015. 
30. Waldeland E, Malinen E. Review of the dose-to-water energy dependence of alanine and lithium formate EPR dosimeters and LiF TL-dosimeterscomparison with Monte Carlo simulations. Radiat Meas. 2011;46:945-51. https://doi.org/10.1016/j.radmeas.2011.03.014.

31. Kudynski R, Kudynska J, Buckmaster HA. The application of EPR dosimetry for radiotherapy and radiation protection. Appl Radiat Isot. 1993;44:9036. https://doi.org/10.1016/0969-8043(93)90042-9.

32. Rech AB, Barbi GL, Ventura LHA, Guimarães FS, Oliveira HF, Baffa O. In vivo dose evaluation during gynaecological radiotherapy using L-alanine/
ESR dosimetry. Radiat Prot Dosimetry. 2014;159:194-8. https://doi.org/10. 1093/rpd/ncu128.

\section{Publisher's Note}

Springer Nature remains neutral with regard to jurisdictional claims in published maps and institutional affiliations.
Ready to submit your research? Choose BMC and benefit from:

- fast, convenient online submission

- thorough peer review by experienced researchers in your field

- rapid publication on acceptance

- support for research data, including large and complex data types

- gold Open Access which fosters wider collaboration and increased citations

- maximum visibility for your research: over 100M website views per year

At BMC, research is always in progress.

Learn more biomedcentral.com/submissions 